\title{
Design of a Maglev Vibration Test Platform for the Research of Maglev Vehicle-girder Coupled Vibration Problem
}

\author{
Danfeng Zhou , Lianchun Wang, Jie Li and Peichang Yu \\ College of Mechatronics Engineering and Automation, National University of Defense Technology, 410073 Changsha, P.R. China
}

\begin{abstract}
The maglev vehicle-girder coupled vibration problem has been encountered in many maglev test or commercial lines, which significantly degrade the performance of the maglev train. In previous research on the principle of the coupled vibration problem, it has been discovered that the fundamental model of the maglev girder can be simplified as a series of mass-spring resonators of different but related resonance frequencies, and that the stability of the vehicle-girder coupled system can be investigated by separately examining the stability of each massspring resonator - electromagnet coupled system. Based on this conclusion, a maglev test platform, which includes a single electromagnetic suspension control system, is built for experimental study of the coupled vibration problem. The guideway of the test platform is supported by a number of springs so as to change its flexibility. The mass of the guideway can also be changed by adjusting extra weights attached to it. By changing the flexibility and mass of the guideway, the rules of the maglev vehicle-girder coupled vibration problem are to be examined through experiments, and related theory on the vehicle-girder self-excited vibration proposed in previous research is also testified.
\end{abstract}

\section{Introduction}

As the development of the maglev transport around the world, the maglev vehicle-girder coupled vibration problem has been discovered, and it gains more and more attention as more and more maglev test or commercial lines are constructed [1-4]. Recent test run of the Changsha urban maglev line revealed that the resonance is more easily to be encountered when the maglev train is passing through some steel girders at low speed. Similar problem has also been encountered in the Tangshan maglev test line. In [5], the coupled vibration problems in the EMS (Electromagnetic Suspension) maglev train are reviewed. In [6], the principle of the maglev vehiclegirder self-excited vibration problem was investigated in the frequency domain, and some important conclusions were drawn, but without experimental validation.

On a commercial maglev line, the parameters of the girders, such as the mass and the rigidness, are fixed and they are difficult to change in the presence of maglev vehicle-girder self-excited vibration problem; therefore, experimental study on a commercial line are quite limited to the given conditions. To overcome this problem, based on the maglev vehicle-girder coupled model developed in previous research, a maglev vibration test platform is built for experimental study of the self-excited vibration problem. The test platform equipped with a single electromagnet levitation control system and a flexible guideway. To best imitate the theoretical model of the maglev vehicle-girder coupled system, the levitation control system is equipped with the same sensors as a real maglev train does. As an approximation of the flexible girder in a maglev line, the guideway of the test platform is designed as a rigid beam supported by elastic springs. The mass of the rigid beam, as well as the elasticity of the springs, can be adjusted so as to study the stability of the coupled system under different girder conditions. This configuration provides a wide range of girder parameters which are benefit for the study of the conditions when the maglev vehicle-girder self-excited vibration problem may occur, and it also provides an approach to seek control algorithms that is useful to suppress the maglev vehiclegirder self-excited vibration through experiments.

\section{Modelling of the maglev vehicle-girder coupled system}

As shown in Fig. 1, the maglev vehicle-girder selfexcited vibration generally occurs when a maglev vehicle is passing through an elevated girder at a very low speed, in which condition many levitation control units are capable of sensing the vertical displacement of the girder and adjusting their levitation currents thereafter. For some reason, the adjustments of the levitation currents form a consultant force which enlarges the displacement of the girder, resulting in an increasing vibration of the girder, which is called self-excited vibration (or resonance in some literature). It is believed that the selfexcited vibration is partly caused by the flexibility of the girder, hence the displacement of the girder subject to external forces are investigated first. 


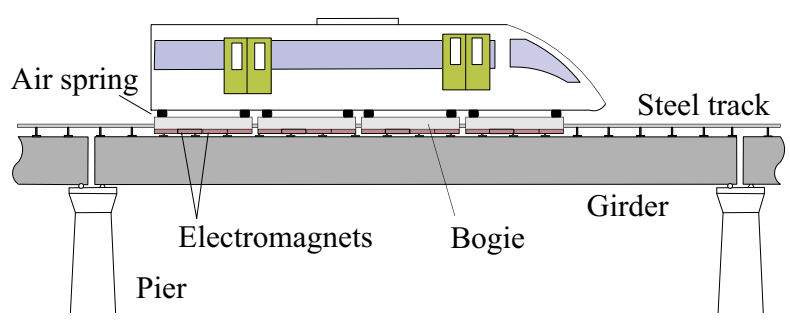

Figure 1. Schematic of a maglev vehicle passing through an elevated girder.

Given the span length of the girder, $L$, the mass per unit length and the bending stiffness of the bridge, $\rho$ and $E I$, respectively, the displacement of the girder, $y(x, t)$, can be described by the following equation:

$$
E I \frac{\partial^{4} y(x, t)}{\partial x^{4}}+\rho \frac{\partial^{2} y(x, t)}{\partial t^{2}}=f(x, t),
$$

where $f(x, t)$ is the distributed force acting on the girder. For a maglev girder, it can be treated as a Bernoulli-Euler beam, and the vibratory motion of the beam can be decomposed into the superposition of all mode shapes; namely,

$$
y(x, t)=\sum_{n=1}^{\infty} y_{n}(t) \phi_{n}(x),
$$

where $\phi_{n}(x)$ is the $n$-th order modal shape, which is a combination of $\sin \left(\lambda_{n} x\right), \cos \left(\lambda_{n} x\right), \sinh \left(\lambda_{n} x\right)$ and $\cos \left(\lambda_{n} x\right)$; $y_{n}(t)$ is the generalized displacement of the $n$-th order modal shape. The coefficients and $\lambda_{n}$ in $\phi_{n}(x)$ are determined by the boundary conditions of the beam. Substituting Eq. (2) into Eq. (1), multiplying both sides of the resultant equation by $\phi_{n}(x)$, and integrating both sides along the beam, gives:

$$
\begin{array}{r}
\lambda_{n}^{4} y_{n}(t) \frac{E I}{\rho} \int_{0}^{L} \phi_{n}^{2}(x) \mathrm{d} x+\ddot{y}_{n}(t) \int_{0}^{L} \phi_{n}^{2}(x) \mathrm{d} x \\
=\frac{1}{\rho} \int_{0}^{L} f(x, t) \phi_{n}(x) \mathrm{d} x
\end{array}
$$

Note that the mode orthogonality condition for linear beams has been applied in the computation. Eq. (3) can be rewritten in another form:

$$
\ddot{y}_{n}(t)+\omega_{n}^{2} y_{n}(t)=\frac{\bar{F}_{n}(t)}{M_{n}},
$$

where

$$
\begin{aligned}
& M_{n}=\rho \int_{0}^{L} \phi_{n}^{2}(x) \mathrm{d} x \\
& \bar{F}_{n}(t)=\int_{0}^{L} f(x, t) \phi_{n}(x) \mathrm{d} x \\
& \omega_{n}^{2}=\frac{E I \lambda_{n}^{4}}{\rho}
\end{aligned}
$$

Eq.(4) reveals that the dynamic response of the beam under external force excitations is equivalent to the superposition of the responses of a series of mass-spring systems under generalized force excitations. Compared with the length of the elevated girder, the length of the electromagnetic forces can be neglected, and they can be approximately treated as point contact forces. For the $k$-th electromagnetic force, $F_{k}$, we have

$$
f_{k}(x, t)=F_{k}(t) \delta\left(x-x_{k}\right),
$$

where $\delta(\cdot)$ is the Dirac delta function; and $x_{k}$ corresponds to the location of the $k$-th electromagnetic force. Consequently,

$$
\bar{F}_{n, k}(t)=\int_{0}^{L} f(x, t) \phi_{n}(x) \mathrm{d} x=F_{k}(t) \phi_{n}\left(x_{k}\right) .
$$

Substituting Eq.(10) into Eq.(5), the following equation can be obtained:

$$
\ddot{y}_{n}(t)+\omega_{n}^{2} y_{n}(t)=\frac{F_{k}(t) \phi_{n}\left(x_{k}\right)}{M_{n}} .
$$

It may be noticed that the dynamic equation for a mass-spring resonator is

$$
\ddot{y}(t)+\omega^{2} y(t)=\frac{F(t)}{m},
$$

where $m$ is the mass of the resonator, and $\omega$ is the natural frequency of the resonator. Comparing Eq.(11) with Eq.(10), it can be seen that the dynamic behavior of the elevated girder subject to an electromagnetic force is equivalent to the superposition of the responses of infinitely many mass-spring resonators under the excitation of the same force, as demonstrated in Figure 2.

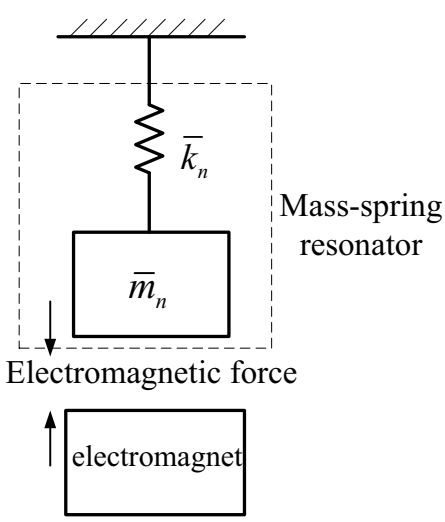

Figure 2. Model of a mass-spring resonator.

The equivalent mass and rigidness of the $n$-th resonator can be given by

$$
\begin{aligned}
& \bar{m}_{n}=\frac{M_{n}}{\phi_{n}\left(x_{k}\right)}, \\
& \bar{k}_{n}=\frac{M_{n} \omega_{n}^{2}}{\phi_{n}\left(x_{k}\right)} .
\end{aligned}
$$


Since $\phi_{n}\left(x_{k}\right)$ is not a constant, Eqs.(12) and (13) gives a hint that the mechanical impedance of the girder is not a constant, either, but a variable depending on the location of the electromagnetic force. For example, the displacement of a simply supported beam can be described as a superposition of sinusoidal functions:

$$
y(x, t)=\sum_{n=1}^{\infty} y_{n}(t) \sin \frac{n \pi x}{L} .
$$
is:

The equivalent mass of the $n$-th mass-spring resonator

$$
\bar{m}_{n}=\frac{\rho L}{2} \csc \frac{n \pi x_{0}}{L}
$$

Here, $\csc (\cdot)$ is the cosecant function. Consequently, the equivalent stiffness of the SDOF resonator can also be obtained, which is:

$$
\bar{k}_{n}=\frac{E I}{2}\left(\frac{n \pi}{L}\right)^{4} \csc \frac{n \pi x_{0}}{L} .
$$

By analogy to the SDOF system, the mechanical impedance of the $n$-th vibration mode of the girder can be defined as:

$$
Z_{n}=\sqrt{\bar{k}_{n} \bar{m}_{n}}=\frac{\sqrt{E I \rho L}}{2}\left(\frac{n \pi}{L}\right)^{2} \csc \frac{n \pi x_{0}}{L} .
$$

Eq.(17) shows that the mechanical impedance of the girder has a lower bound, which corresponds to the situation when the electromagnetic force is at the location where the displacement of the mode shape reaches its maximal value. Furthermore, the mechanical impedance of the girder is proportional to $n^{2}$, which indicates that the higher order the vibration mode is, the larger becomes the mechanical impedance, and the less likely the coupled vibration will occur. This explains why the maglev vehicle-girder self-excited vibration generally occurs at the fundamental order mode of the girder. For a full scale maglev train, the electromagnetic forces are distributed at different locations along the girder, thus the mechanical impedance for each electromagnet is different from others. In this way, the stability of the vehicle-girder coupled system can be deemed as a result of separated electromagnetic forces acting on different size of massspring resonators. If most of the electromagnet-resonator coupled systems are stable, the corresponding vehiclegirder coupled system would be stable; otherwise, the vehicle-girder coupled system would be unstable.

As a basic unit of the vehicle-girder coupled model, the investigation of a single electromagnet-resonator coupled system is essentially important to the theoretical analysis of the stability of the vehicle-girder coupled system. This work has been reported in previous research [6], yet has not been verified experimentally due to the limit of conditions on a real maglev test line. Therefore, the purpose of this paper is to validate the theoretical conclusions by setting up a small scale experimental platform which includes a levitation control system and a mass-spring resonator corresponding to a lower vibration mode of the girder. Compared with the full-scale maglev train, the small scale platform is convenient to change the track and levitation parameters, and to obtain a wider range of parameters, which will benefit for the validation of the theoretical analysis under some extreme conditions.

\section{Design of the maglev vibration test platform}

As discussed in the previous section, the maglev vehicleguideway coupled test platform should include a massspring resonator and a levitation control system. The schematic, which demonstrates the basic structure of the platform, is shown in Fig. 3, in which it can be seen that this platform consists of an electromagnet and a segment of track which is supported by springs. To keep balance, the track is supported by two springs located at each terminal of the beam; therefore, the mass of the track and the flexibility of the springs make up of a mass-spring resonator in vertical direction. Since the rigidness of the beam is much larger compared with the rigidness of the springs, the flexibility of the beam can be neglected, and the natural frequency of the mass-spring resonator can be approximated calculated by

$$
\omega_{0}=\sqrt{\frac{2 k_{s}}{m}},
$$

where $k_{s}$ is the rigidness of a single spring, $m$ is the mass of the track.

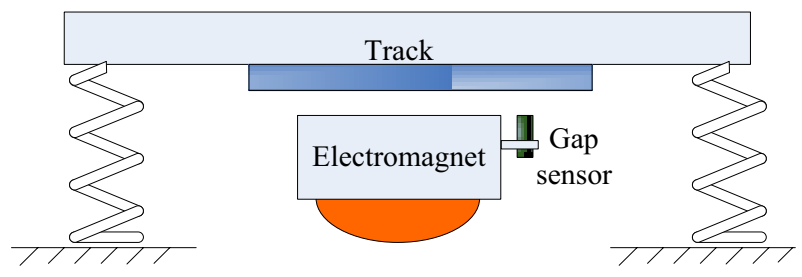

Figure 3. Schematic of the vehicle-guideway coupled vibration test platform.

It may be noticed that the structural of the track system in Fig. 3 cannot keep balance since the rail may swing horizontally. To solve this problem, a guiding gear in vertical direction is needed. Moreover, a guiding and supporting mechanism is also required to guide the movement of the electromagnet, and to support the electromagnet when the equipment is not suspending. A photo of the designed platform is shown in Figure 4, in which the guiding system and the supporting system are considered.

For a real maglev system, the damping of the girder plays an important role on the stability of the vehiclegirder coupled system, hence the damping of the track in the test platform should also be considered. To meet this requirement, several small dampers, whose damping can be adjusted by a screw, are fixed to the track. 


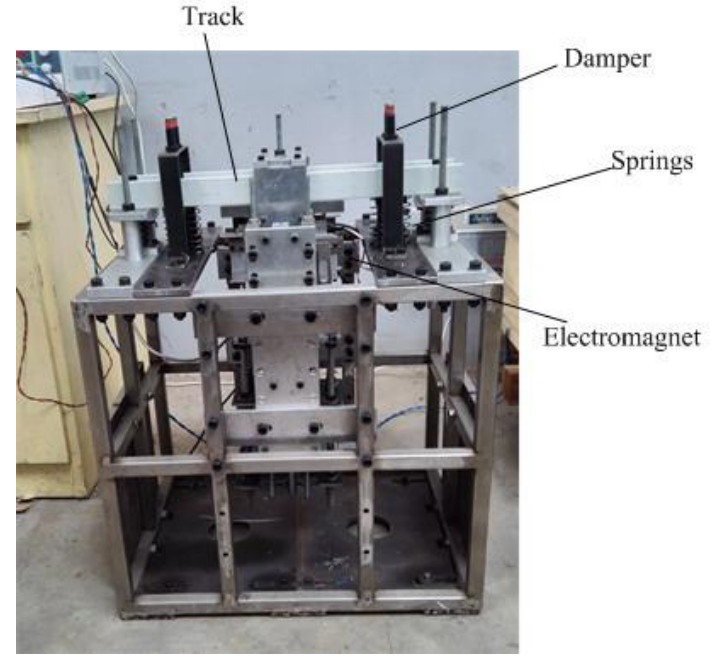

Figure 4. Produced test platform.

To adjust the stiffness of the mass-spring resonator, more springs can be applied to the track to enhance the stiffness of the resonator in vertical direction. Similarly, to adjust the mass of the mass-spring resonator, extra weights (steel blocks) can be attached to the track. Increasing the mass of the track and the stiffness of the springs simultaneously would increase the mechanical impedance of the track. These adjustments can also guarantee that the frequency of the resonator is able to cover a rather wide range so as to simulate different guideway conditions.

A single electromagnet is designed for the levitation control system, and the material of the iron core is the same as that used in a real maglev train. But the material of the coil is enamelled copper wire, rather than aluminium wires used in a real maglev train. Some key parameters of the test platform are listed in Table 1.

Table 1. Key parameters of the designed platform.

\begin{tabular}{|c|c|c|}
\hline Parameter & Value & Unit \\
\hline Mass of track & 5.4 & $\mathrm{~kg}$ \\
\hline Mass of suspension system & 8.02 & $\mathrm{~kg}$ \\
\hline Mass of small weight & 0.33 & $\mathrm{~kg}$ \\
\hline Mass of big weight & 0.99 & $\mathrm{~kg}$ \\
\hline $\begin{array}{c}\text { Area of electromagnetic } \\
\text { pole }\end{array}$ & 0.001 & $\mathrm{~m}^{2}$ \\
\hline Number of coil & 843 & - \\
\hline
\end{tabular}

To achieve stable levitation, a gap sensor is essential to measure the air gap between the rail and the electromagnetic poles; therefore, an eddy-current gap sensor is mounted to one side of the electromagnet and it moves along with the electromagnet. Besides, an accelerometer, which is used to measure the acceleration of the electromagnet in vertical direction, is also mounted to the other side of the electromagnet. The electromagnet is driven by a PWM chopper and is controlled by a digital micro-processor. To study the influence of the control voltage on the stability of the coupled system, the voltage of the chopper is provided by an adjustable power supply with $60 \mathrm{~V}$ maximum output.

\section{Test result of the platform}

\subsection{Frequency range test of the track}

In a real maglev system, the fundamental frequency of the elevated girder is generally below $16 \mathrm{~Hz}$, and the fundamental frequency of the concrete girder is generally lower than that of the steel girder. For example, the fundamental frequencies of the girders in the Tangshan maglev test line have been measured, and it is found that the frequency of the concrete girder is around $9 \mathrm{~Hz}$ for the $18-\mathrm{m}$ long concrete girder, while it is over $13 \mathrm{~Hz}$ for the steel girder of the turnover. To be close to reality, the frequency range of the track in the test platform should cover the frequency range of the real girders. Since the frequency of the track can be lower down by attaching weights onto it, the highest natural frequency should be considered first. Fig. 5(a) shows the free vibration of the track by knocking it with a hammer, which appears that the attenuation ratio of the vibration resembles that of a concrete girder. Figure 5(b) shows the power spectrum density (PSD) of the gap signal when the track is repeatedly knocked. It can be seen that natural frequency of the track without additional weight is $18.4 \mathrm{~Hz}$, which is higher than the fundamental frequency of the real steel girders. If higher natural frequency is required, additional springs can be applied to increase the stiffness of the track.
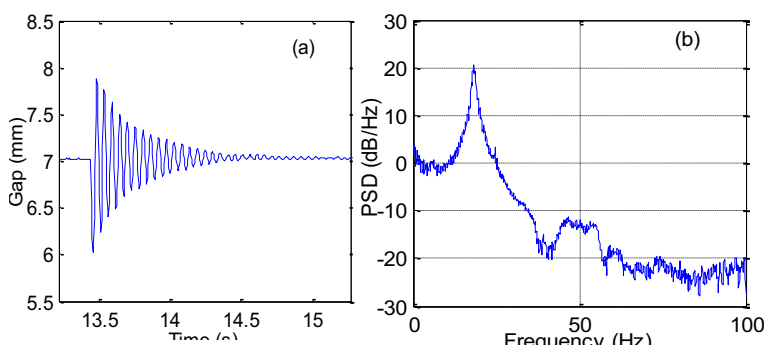

Figure 5. Free vibration and PSD of the track.

The natural frequency of the track can also be decrease by attaching additional weights to the rail. Field tests show that the natural frequency of the track decreases to $16.4 \mathrm{~Hz}$ when 3 small weights are attached to each side of the track, and the frequency drops down to $12.0 \mathrm{~Hz}$ when $4 \mathrm{big}$ weights are added to each side of the track. Lower frequency can also be achieved by adding more weights to the track.

To investigate the differences of the steel girder and the concrete girder, the dampers in the test platform can be adjusted so as to obtain required damping ratio of the track.

\subsection{Stability test of the coupled system}

In the previous research on the principle of the maglev vehicle-girder self-excited vibration problem [6], the frequency characteristics of the levitation system and the girder were separately analyzed, and the stability of the coupled system was investigated by the open-loop system which joins these two subsystems together. The 
Nyquist stability theory was employed as the basic stability criterion. The investigation drew a conclusion that if the self-excited vibration occurs, the frequency of the vibration is determined by both the unstable mode frequency of the girder and the critical frequency of the levitation system. The latter, which was defined in the literature [6], corresponds to the frequency when the phase response of the levitation system crosses the $-180^{\circ}$ line downwards. Theoretically, the frequency of the selfexcited vibration is higher than either of these two frequencies mentioned above (conclusion 1).

Another conclusion had also been drawn in [6] that when the mode frequency of the girder is lower than the critical frequency of the levitation system, the selfexcited vibration is unlikely to occur; otherwise, the selfexcited vibration will sure to occur when the damping of the track is neglected. However, when the damping of the track is taken into account, the self-excited vibration only occurs when the mode frequency of the girder is close to the critical frequency of the levitation system (conclusion 2).

In this section, these conclusions will be tested through a series of experiments in the platform. It has been tested in the previous section that the natural frequency of the test platform covers the interested frequency range for a real maglev girder, thus the experiments will be organized by changing the natural frequency of the track at each time. The experiments starts under the condition when the track is free of extra weights, and followed by the decrease of the track natural frequency by adding some weights on the track at each time. The test results will then be listed in a table for comparison, so as to testify the conclusions mentioned above.

Fig. 6 shows the test result of the case when no extra weights are added. It can be shown that after a short period of time, the levitation begins to oscillate, and selfexcited vibration occurs afterwards. Investigation of the track indicates that under such a circumstance, the natural frequency of the track is $18.4 \mathrm{~Hz}$, which is quite high compared with the critical frequency of an ordinary levitation system, thus self-excited vibration tends to occur. Fig. 6(b) shows the corresponding PSD of the levitation gap, which shows that the frequency of the selfexcited vibration reaches $20.7 \mathrm{~Hz}$, which is higher than the natural frequency of the track. This agrees with the conclusion 1 mentioned above.
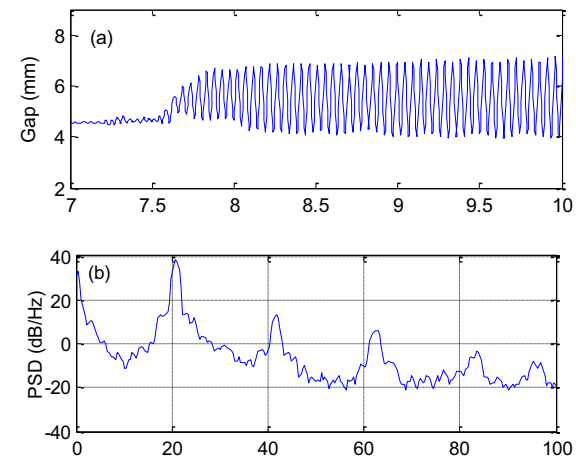

Figure 6. Levitation gap and its corresponding PSD when no extra weights are attached to the track.
Other tests under different track conditions are listed in Table 2, and the stability of these tests are also presented therein. It should be noticed that the values of the vibration frequency are obtained by power spectrum analysis of the levitation gap, regardless of the stability of the coupled system. The vibration frequency can be identified even for the stable cases, which may arise from external disturbances which cause vibratory movements.

It can be seen that as the frequency of the track lowers down, the coupled system becomes stable, this may agree with conclusion 2 presented above. It can also be seen that all the vibration frequencies are higher than the natural frequencies of the track. Therefore, conclusion 1 is also validated.

Table 2. Test results under different track conditions.

\begin{tabular}{|c|c|c|c|c|}
\hline $\begin{array}{c}\text { Track freq. } \\
(\mathrm{Hz})\end{array}$ & $k_{p}$ & $k_{d}$ & $\begin{array}{c}\text { Vibration } \\
\text { freq. (Hz) }\end{array}$ & Stability \\
\hline 12.0 & 40 & 50 & 13.7 & Stable \\
\hline 12.0 & 60 & 50 & 15.2 & Stable \\
\hline 12.0 & 80 & 60 & 15.6 & Stable \\
\hline 13.2 & 60 & 60 & 15.7 & Stable \\
\hline 15.8 & 50 & 50 & 17.1 & Stable \\
\hline 15.8 & 70 & 70 & 17.8 & Stable \\
\hline 16.4 & 60 & 60 & 19.0 & Stable \\
\hline 18.4 & 60 & 60 & 20.7 & Unstable \\
\hline
\end{tabular}

Table 2 also gives a hint that the control parameters also affects the frequency of the self-excited vibration, which is a topic to be discussed in further study.

\section{Conclusions}

The maglev vehicle-girder coupled vibration problem has been encountered in many maglev test or commercial lines, which significantly degrade the performance of the maglev train. This paper presents a methodology to study the principle of the coupled vibration problem by designing a small test platform. The results show that the equipment can well be used to study the fundamental model the vehicle-guideway coupled system. Using this platform, some theoretical results proposed previously are also testified, which shows that the designed platform is a good supplement for the study of the maglev vehiclegirder coupled vibration problem.

\section{Acknowledgement}

This work was supported by the National Key Technology R\&D Program of the 12th Five-year plan (No. 2012BAG07B01-13), and National Nature and Science Foundation of China (No. 11302252).

\section{References}

1. H. Wang, X. Zhong, G. Shen, Journal of Vibration and Control. 21, 408-416 (2015)

2. J.S. Lee, S.D. Kwon, M.Y. Kim, et al., Journal of Sound and Vibration. 328, 301-317 (2009) 
3. B.M. Jin, I.G. Kim, Y.J. Kim, et al., International Conference on Electrical Machines and Systems; Seoul, Korea. 1959-1962 ( 2007)

4. K.J. Kim, J.B.Han, H.S. Han, et al., Vehicle System Dynamics.53, 587-601 ( 2015)

5. D.F. Zhou, C. H. Hansen, J. Li, et al., International Journal of Acoustics and Vibration. 15, 10-23 (2010)

6. D.F. Zhou, C.H. Hansen, J. Li, Journal of Sound and Vibration. 330, 883-901 (2011) 